\title{
Metastasis of Oropharyngeal Carcinoma to the Site of Percutaneous Endoscopic Gastrostomy: Literature Review
}

\author{
Radwan Kassiri, c, Alaric Cavaille ${ }^{\mathrm{a}}$, Gabriele Barabino ${ }^{\mathrm{a}}$, \\ Jean-Marc Dumollard ${ }^{b}$, Jack Porcheron ${ }^{\mathrm{a}}$
}

\begin{abstract}
Complications of percutaneous endoscopic gastrostomy (PEG) are relatively uncommon. PEG site metastases are iatrogenic complications of PEG tube placement. The literature search revealed only a few descriptions of tumor implantation at the PEG site. The patient is a 62-year-old male with a history of alcoholism and tobacco abuse. He was diagnosed with stage IV (T4 N2M0) squamous cell carcinoma (SCC) of the piriform recess. A PEG tube was placed and he underwent wide resection of aerodigestive tract. One year later, the patient mentioned a granulation tissue forming around the tube site. An en bloc resection of the abdominal wall was performed. The postoperative histopathological findings were in accordance with the diagnosis of SCC. Herein, an extremely rare case of metastasis of an oropharyngeal cancer at a PEG stoma is described. Multiple theories of metastatic spread have been proposed. The mean time to PEG site implantation was 8 months after insertion. In patients of whom the interval between PEG placement and diagnosis of metastasis was 1 year, Douglas et al concluded that hematogenous spread is less likely than direct implantation of cells. The exact mechanism of abdominal wall metastasis in our patient still remains unclear. In our case, the time to PEG site implantation was 1 year and lymph nodes were invaded before PEG placement (T4 N2M0). Metastatic cancer should be evoked when skin changes at the PEG site in patients with head-and-neck cancer.
\end{abstract}

Keywords: Surgery; Percutaneous endoscopic gastrostomy; Squamous cell carcinoma; Head-and-neck cancer

Manuscript accepted for publication February 19, 2014

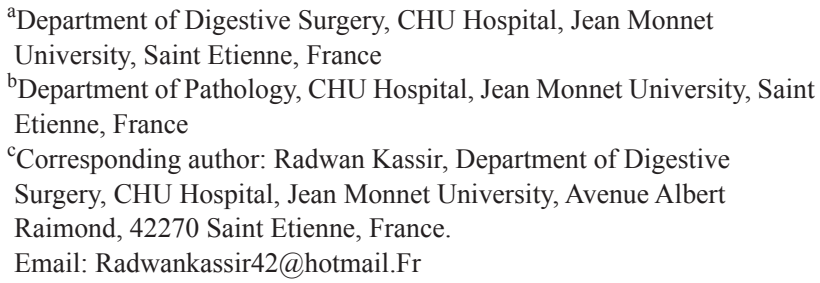

doi: http://dx.doi.org/10.14740/jcs223w

\section{Introduction}

Patients with oropharyngeal carcinoma often need a percutaneous endoscopic gastrostomy (PEG) to provide adequate nutrition because of the inability to swallow before or after surgery and adjuvant therapies. Complications of PEG are relatively uncommon, but include local infection, peritonitis, tube blockage, tube dislodgement, gastroesophageal reflux and aspiration pneumonia. However, metastasis of the original tumor to the gastrostomy exit site may occur. We report a case of tumor implantation at the PEG site from squamous cell cancer (SCC) of the head and neck.

\section{Case Report}

The patient is a 62-year-old male with a history of alcoholism and tobacco abuse. He had no personal or family medical or surgical history and had no history of medical drug use. He was diagnosed with stage IV (T4 N2M0) SCC of the piriform recess, epiglottic vallecula and base of the tongue. At the time of the original surgery, a PEG tube was placed to circumvent anticipated difficulties in swallowing after surgery. The pull-through method of gastrostomy tube placement had been used in our patient. He underwent wide resection that included the piriform recess, epiglottic vallecula and about $50 \%$ of the base of the tongue. He also underwent a neck dissection. To cover the defect, a muscle free flap from the pectoralis major was utilized. The postoperative period was uncomplicated. At microscopic examination, all surgical margins were free of tumor involvement. He had two positive nodes. The patient subsequently underwent a radiation therapy.

One year later, the patient mentioned a granulation tissue forming around the tube site. The mass reached a size of $70 \mathrm{~mm}$ in diameter. CT scan of the abdomen showed a large mass $(45 \times 38 \times 70 \mathrm{~mm})$ extending through the abdominal wall and the gastrostomy exit site. Metastatic work-up to include CT of the head and bone scan was negative. An en bloc resection of the abdominal wall was performed. The postoperative histopathological findings were in accordance 


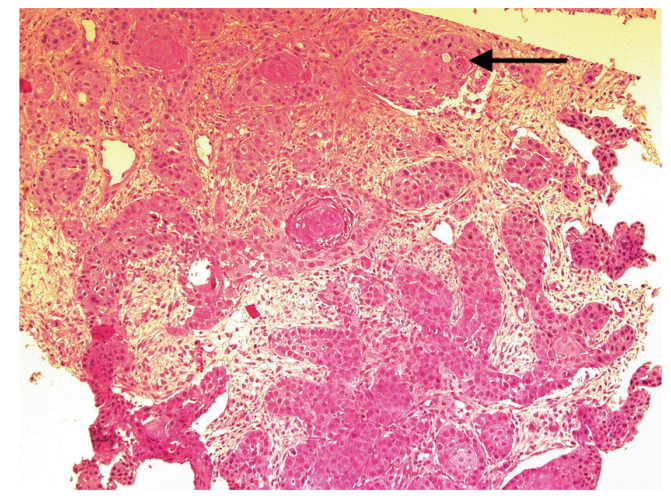

Figure 1. The anatomopathologic results (HES $\times 10$ original magnification): squamous cell carcinoma. Arrow: dyskeratosis.

with the diagnosis of SCC (Fig. 1, 2). It was to consider that this tumor came from the patient's original neck cancer. The resection margin did not contain any malignant cells. The postoperative period was uncomplicated. The patient is alive 3 years after the surgery without evidence of recurrence.

\section{Discussion}

In 1980, Gauderer et al first described the technique of PEG [1]. PEG is a pillar in intrajejunal feeding for patients with obstructive oropharyngeal cancer or undernourished. Complications of PEG include local infection, peritonitis, tube blockage, tube dislodgement, dyspnea, gastroesophageal reflux and aspiration pneumonia [2]. PEG site metastases are iatrogenic complications of PEG tube placement. The incidence of this complication is not known. The literature search revealed only a few descriptions of tumor implantation at the PEG site and these are limited to clinical case reports. The oropharynx was the most common primary tumor site, followed by hypopharynx, oral cavity and larynx [3]. Herein, an extremely rare case of metastasis of an oropharyngeal cancer at a PEG stoma is described. This case has been compared with similar cases reported in the literature.

The first case of tumor implantation at the PEG site from SCC of the head and neck was reported in 1989 [4]. After literature review, the mean time to PEG site implantation was 8 months after insertion. But the time of tumor implantation at the PEG site from SCC of the head and neck can vary between 1 and 24 months. Most patients with PEG site metastasis present with vague abdominal discomfort, unexplained skin changes, persistent stomal drainage or constipation [5]. Presentations of PEG site metastasis include also incidental imaging findings. Early detection may provide a chance of cure.

Multiple theories of metastatic spread have been proposed, which include the shedding of tumor cells into the gastrointestinal tract from the original head-and-neck cancer

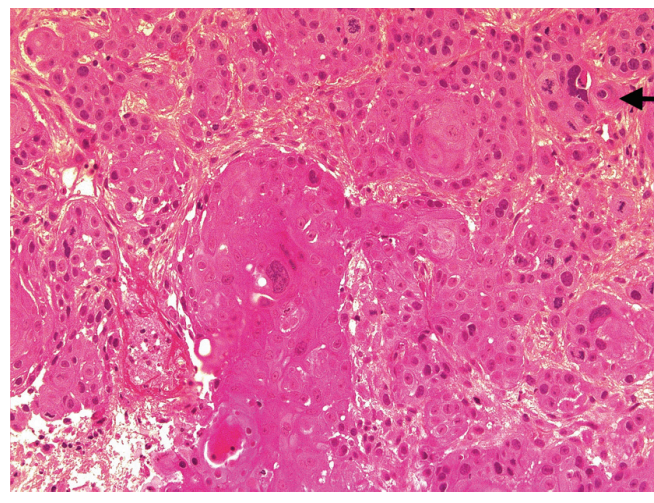

Figure 2. The anatomopathologic results (HES $\times 20$ original magnification): squamous cell carcinoma. Arrow: dyskeratosis.

and the direct implantation at the time of PEG placement where instruments have injured the tissue. The shedding of tumor cells into the gastrointestinal tract from the original head-and-neck cancer is possible because of direct contact between tumor cells and the gastrostomy tube. The direct implantation at the time of PEG placement may occur through lymphatic spread and hematogenous. In patients of whom the interval between PEG placement and diagnosis of metastasis was 1 year, Douglas et al concluded that hematogenous spread is less likely than direct implantation of cells [6]. The exact mechanism of abdominal wall metastasis in our patient still remains unclear. In our case, the time to PEG site implantation was 1 year and lymph nodes were invaded before PEG placement (T4 N2M0). Therefore, direct implantation of cells is not the only hypothesis to explain this complication in our case.

In head-and-neck cancer patients, special precautions must be taken during the PEG. So, before PEG tube placement, meticulous evaluation of the oropharynx and hypopharynx is advised. Raynor et al demonstrated that PEG tube placement after tumor resection has the lowest incidence of postoperative complications [7]. Selz et al demonstrated that "PEG can be performed by the otolaryngologist-head and neck surgeon with minimal or no morbidity at the time of staging or definitive procedure" [8]. When PEG placement is required, the push method is recommended. The push PEG technique has a significantly lower risk of complications compared with the pull technique [9].

Rustom et al concluded that the PEG tube is superior to the radiologically inserted gastrostomy and surgically inserted gastrostomy because this procedure had fewer complications and was safer [10]. But we believe that the gastrostomy tube placement by laparoscopy or laparotomy is minimally invasive method and may be more appropriate in head-and-neck cancer patients. These methods avoid tumorcontaminated fields. Therefore, the tumor implantation at the PEG site in patients with active aerodigestive tract malignancies should be avoided using these methods of establishing 
enteral access.

Metastatic cancer should be evoked when skin changes at the PEG site in patients with head-and-neck cancer. The "push" technique of PEG tube placement and the gastrostomy tube placement by laparoscopy or laparotomy can avoid direct implantation of malignant cells into an enteral access site.

\section{Conflict of Interest}

No.

\section{References}

1. Gauderer MW, Ponsky JL, Izant RJ, Jr. Gastrostomy without laparotomy: a percutaneous endoscopic technique. J Pediatr Surg. 1980;15(6):872-875.

2. Anath $\mathrm{S}$, Amin M. Implantation of oral squamous cell carcinoma at the site of a percutaneous endoscopic gastrostomy: a case report. Br J Oral Maxillofacial Surg. 2002;40:25-30.

3. Huang AT, Georgolios A, Espino S, Kaplan B, Neifeld J, Reiter ER. Percutaneous endoscopic gastrostomy site metastasis from head and neck squamous cell carcinoma: case series and literature review. J Otolaryngol Head Neck Surg. 2013;42:20.

4. Preyer S, Thul P. Gastric metastasis of squamous cell carcinoma of the head and neck after percutaneous endoscopic gastrostomy--report of a case. Endoscopy. 1989;21(6):295.

5. Cappell MS. Risk factors and risk reduction of malignant seeding of the percutaneous endoscopic gastrostomy track from pharyngoesophageal malignancy: a review of all 44 known reported cases. Am J Gastroenterol. 2007;102(6):1307-1311.

6. Douglas JG, Koh W-J, Laramore GE. Metastasis to a percutaneous gastrostomy site from head and neck cancer: radiobio-logic considerations. Head Neck. 2000;22:826830.

7. Raynor EM, Williams MF, Martindale RG, Porubsky ES. Timing of percutaneous endoscopic gastrostomy tube placement in head and neck cancer patients. Otolaryngol Head Neck Surg. 1999;120(4):479-482.

8. Selz PA, Santos PM. Percutaneous endoscopic gastrostomy. A useful tool for the otolaryngologist-head and neck surgeon. Arch Otolaryngol Head Neck Surg. 1995;121(11):1249-1252.

9. Tucker AT, Gourin CG, Ghegan MD, Porubsky ES, Martindale RG, Terris DJ. 'Push' versus 'pull' percutaneous endoscopic gastrostomy tube placement in patients with advanced head and neck cancer. Laryngoscope. 2003;113(11):1898-1902.

10. Rustom IK, Jebreel A, Tayyab M, England RJ, Stafford ND. Percutaneous endoscopic, radiological and surgical gastrostomy tubes: a comparison study in head and neck cancer patients. J Laryngol Otol. 2006;120(6):463-466. 